\title{
Non-hyperbolic ergodic measures with large support
}

\author{
Ch. Bonatti, L. J. Díaz, and A. Gorodetski
}

November 3, 2018

\begin{abstract}
We prove that there is a residual subset $\mathcal{S}$ in $\operatorname{Diff}^{1}(M)$ such that, for every $f \in \mathcal{S}$, any homoclinic class of $f$ with invariant one dimensional central bundle containing saddles of different indices (i.e. with different dimensions of the stable invariant manifold) coincides with the support of some invariant ergodic non-hyperbolic (one of the Lyapunov exponents is equal to zero) measure of $f$.
\end{abstract}

\section{Introduction}

How to characterize the absence of uniform hyperbolicity? What dynamical structures can not exist in the uniformly hyperbolic setting but must be present in the complement? In this paper, we study how the non-hyperbolicity is detected in the ergodic level. Namely, we consider non-hyperbolic invariant measures as indication of non-hyperbolicity, and construct such measures with full support for partially hyperbolic homoclinic classes.

Some other candidates for the role of "non-hyperbolic structure" are the cycles (homoclinic tangencies and heterodimensional cycles), super-exponential growth of the number of periodic points [24, 7], absence of shadowing property [9, 37, 3, 34], and non-existence of symbolic extensions [18, 4, 16].

Keywords: dominated splitting, homoclinic class, Lyapunov exponent, partial hyperbolicity, heterodimenional cycle, support of an invariant measure.

MSC 2000: 37C05, 37C20, 37C29, 37D25, 37D30. 
Recall that if $\mu$ is an ergodic measure of a diffeomorphism $f: M \rightarrow$ $M, \operatorname{dim} M=\mathbf{m}$, then there is a set $\Lambda$ of full $\mu$-measure and real numbers $\chi_{\mu}^{1} \leq \chi_{\mu}^{2} \leq \cdots \leq \chi_{\mu}^{\mathbf{m}}$ such that, for every $x \in \Lambda$ and every non-zero vector $v \in T_{x} M$, one has $\lim _{n \rightarrow \infty} 1 / n \log \left\|D f^{n}(v)\right\|=\chi_{\mu}^{i}$ for some $i=1, \ldots, \mathbf{m}$, see [30, 26]. The number $\chi_{\mu}^{i}$ is the $i$-th Lyapunov exponent of the measure $\mu$.

Definition 1.1. An ergodic invariant measure of a diffeomorphism is called non-hyperbolic if at least one of its Lyapunov exponents is equal to zero.

Inspired by Palis' density conjecture ([31]), we stated the following ([15]):

Conjecture 1. In $\operatorname{Diff}^{r}(M), r \geq 1$, there exists an open and dense subset $\mathcal{U} \subset$ $\operatorname{Diff}^{r}(M)$ such that every diffeomorphism $f \in \mathcal{U}$ is either uniformly hyperbolic or has an ergodic non-hyperbolic invariant measure.

We consider the question of the existence of non-hyperbolic measures for homoclinic classes. The homoclinic class of a hyperbolic periodic point $P$ of a diffeomorphism $f$, denoted by $H(P, f)$, is the closure of the transverse intersections of the invariant manifolds of the orbit of $P$, see [29]. A homoclinic class is a transitive set (there exists a dense orbit) where periodic points also form a dense subset. Note that a homoclinic class may fail to be hyperbolic and may contain saddles of different s-indices (dimension of the stable bundle). This is precisely the setting we consider in this paper. In many important cases homoclinic classes are used to structure the global dynamics, playing a role similar to that of the basic sets in the hyperbolic theory (in fact, basic sets are a special case of homoclinic classes). In [15] we show the following:

Theorem. There is a residual subset $\mathcal{S}$ of $\operatorname{Diff}^{1}(M)$ such that, for every $f \in \mathcal{S}$, any homoclinic class of $f$ containing saddles of different $\mathrm{s}$-indices (dimension of the stable bundle) contains also an uncountable support of an invariant ergodic non-hyperbolic measure of $f$.

In view of this result, it is natural to consider the following question about the support of the ergodic non-hyperbolic measures in non-hyperbolic homoclinic class.

Question 1. When does a non-hyperbolic homoclinic class equal to the support of a non-hyperbolic ergodic measure?

The main result of this paper is the following (for an accurate formal statement see Theorem 1): 
Main result. For a $C^{1}$-generic diffeomorphism $f$, every homoclinic class $H$ with a 1-dimensional central direction and saddles of different indices is the support of an ergodic non-hyperbolic invariant measure $\mu$ of $f, H=\operatorname{supp} \mu$.

In an opposite direction, we would like to mention the results in [1]: for $C^{1}$-generic diffeomorphisms generic measures supported on isolated homoclinic class are ergodic and hyperbolic (all Lyapunov exponents are non-zero).

The constructions in [15] translate to the setting of homoclinic classes the arguments in [21] and [25] about the existence of non-hyperbolic ergodic measures for skew products defined over the circle $\mathbb{S}^{1}$ and the corresponding smooth realizations. There the circle $\mathbb{S}^{1}$ corresponds to the center direction and the fact that the central direction is one-dimensional is essential. This condition can be precisely formulated in terms of dominated splittings.

Definition 1.2 (Dominated splitting and partial hyperbolicity). Consider a diffeomorphism $f$ and a compact $f$-invariant set $\Lambda . A D f$-invariant splitting $T_{\Lambda} M=E \oplus F$ over $\Lambda$ is dominated if the fibers $E_{x}$ and $F_{x}$ of $E$ and $F$ have constant dimension and there is a constant $k \in \mathbb{N}$ such that

$$
\frac{\left\|D_{x} f^{k}(u)\right\|}{\left\|D_{x} f^{k}(w)\right\|}<\frac{1}{2},
$$

for every $x \in \Lambda$ and every pair of unitary vectors $u \in E_{x}$ and $w \in F_{x}$.

In some cases, we consider splittings with three bundles. A Df-invariant splitting

$$
T_{\Lambda} M=E \oplus F \oplus G
$$

over $\Lambda$ is dominated if both splittings $(E \oplus F) \oplus G$ and $E \oplus(F \oplus G)$ are dominated.

The dominated splitting $T_{\Lambda} M=E \oplus F \oplus G$ is partially hyperbolic if $E$ and $G$ are both uniformly hyperbolic and at least one of them is not empty. We say that $F$ is the central direction of the splitting.

In 15] general (non-hyperbolic) homoclinic classes are considered (i.e., there is no hyperbolic-like assumptions). A key step in [15] is to identify a partially hyperbolic region $\Lambda$ of the homoclinic class where the (non-hyperbolic) central direction has dimension one: there is a partially hyperbolic splitting $T_{\Lambda} M=$ $E \oplus F \oplus G$, where $F$ is one-dimensional and non-hyperbolic and $E$ and $G$ are uniformly hyperbolic.

In general, this partially hyperbolic region $\Lambda$ is properly contained in the whole homoclinic class, thus the support of the obtained measure is not the 
whole homoclinic class. Prototypical examples of this case are the Derived from Anosov diffeomorphisms defined on $\mathbb{T}^{3}$ obtained via Hopf bifurcations, see [12, 11, where the homoclinic classes are the whole torus and its dominated splitting is of the form $T \mathbb{T}^{3}=E \oplus E^{\mathrm{u}}$, where $E$ is two-dimensional, non-hyperbolic, and undecomposable (it does not contain one-dimensional invariant directions). Thus in these cases the arguments in [15] do not provide a non-hyperbolic measure supported on the whole homoclinic class.

On the other hand, when the non-hyperbolic central bundle has a onedimensional invariant direction one can prove the following:

Theorem 1. Let $M$ be a closed manifold, $\operatorname{dim} M \geq 3$. There is a residual subset $\mathcal{R}$ of $\operatorname{Diff}^{1}(M)$ such that for every $f \in \mathcal{R}$ and every homoclinic class $H(f)$ of $f$ such that

- $H(f)$ has a dominated splitting $T_{H(f)} M=E \oplus F \oplus G$, where $F$ has dimension one,

- $H(f)$ contains saddles of $\mathrm{s}-$ indices $\operatorname{dim}(E)$ and $\operatorname{dim}(E \oplus F)=\operatorname{dim}(E)+1$,

there is a non-hyperbolic ergodic $f$-invariant measure $\mu_{H(f)}$ such that

$$
\operatorname{supp} \mu_{H(f)}=H(f) \text {. }
$$

The simplest setting for our result is the one of homoclinic classes having a partially hyperbolic splitting with a one-dimensional central direction. There are two main sorts of such homoclinic classes. On the one hand, there are the non-hyperbolic homoclinic classes generated by unfolding heterodimensional cycles, see [14, 17]. On the other hand, there are robustly transitive and non-hyperbolic diffeomorphisms (in this case, the homoclinic class is the whole ambient manifold). Recall that a diffeomorphism $f$ is robustly transitive if there is a neighborhood $\mathcal{U}_{f}$ of $f$ in $\operatorname{Diff}^{1}(M)$ consisting of transitive diffeomorphisms: every $g \in \mathcal{U}_{f}$ has a dense orbit in $M$.

Among the robustly transitive diffeomorphisms with one-dimensional central direction, we mention the Derived from Anosov diffeomorphisms (via saddlenode or fork bifurcations) in [26], the time-one maps of transitive Anosov vector fields, see [5], and the perturbations of products of Anosov diffeomorphisms and maps defined on the circle (skew products), [5, 35, 33]. For these cases, Theorem 1 just can be read as follows: 
Corollary 1. Let $M$ be a closed manifold, $\operatorname{dim} M \geq 3$. There is a residual subset $\mathcal{R}$ of $\operatorname{Diff}^{1}(M)$ such that for every $f \in \mathcal{R}$ and every partially hyperbolic homoclinic class of $f$ having saddles of different indices and an one-dimensional central bundle there is a non-hyperbolic ergodic $f$-invariant measure whose support is the whole homoclinic class.

We next discuss the interplay between the results above, the sorts of dominated splittings of homoclinic classes, and the occurrence of homoclinic tangencies. Consider the subset $\operatorname{HT}^{1}(M) \subset \operatorname{Diff}^{1}(M)$ of diffeomorphisms $f$ having a homoclinic tangency associated to some saddle (i.e., the invariant manifolds of the saddle have some non-transverse intersection). Define the (open) set of diffeomorphisms far from homoclinic tangencies by

$$
\mathrm{FT}^{1}(M)=\operatorname{Diff}^{1}(M) \backslash \overline{\mathrm{HT}^{1}(M)} .
$$

A recent result by Yang states the following dichotomy for $C^{1}$-generic diffeomorphisms $f$ far from homoclinic tangencies (i.e., diffeomorphisms $f$ in a residual subset of $\left.\mathrm{FT}^{1}(M)\right)$ : any homoclinic class of $f$ is either hyperbolic or it supports a non-hyperbolic ergodic measure, see [36]. Note that, in principle, there is the possibility for a generic diffeomorphism $f$ to have non-hyperbolic homoclinic classes such that all saddles have the same s-index. For homoclinic classes of diffeomorphisms in $\mathrm{FT}^{1}(M)$ containing saddles of different indices, Theorem 1 implies that there is an ergodic non-hyperbolic measure whose support is the whole homoclinic class.

Finally, we state a refinement of Theorem 1 about the zero Lyapunov exponents of the non-hyperbolic measure. Consider a homoclinic class $H(f)$ of $f$, its s-index (variation) interval is the interval s-ind $(H(f))=[i, j]$, where $i$ and $j$ are the minimum and the maximum of the s-indices of the periodic points in $H(f)$. The homoclinic class $H(f)$ has index variation if $i<j$.

Corollary 2. There is a residual subset $\mathcal{R}$ of $\operatorname{Diff}^{1}(M)$ such that for every diffeomorphism $f \in F T^{1}(M) \cap \mathcal{R}$, every homoclinic class $H(f)$ of $f$ with $\mathrm{s}$-index variation interval $[i, j]$, and every $k \in[i, j)$ there is an ergodic measure $\mu_{H(f)}^{k}$ whose support is the whole homoclinic class $H(f)$ such that its $k$-th Lyapunov exponent $\chi^{k}\left(\mu_{H(f)}^{k}\right)$ is zero.

Indeed, fix $k \in[i, j)$. Theorem 1.1 in [22] claims that if $H(f)$ is a homoclinic class such that $k, k+1$ belong to the index interval s-ind $(H(f))$ and there is no dominated splitting defined over $H(f)$ of the form $E \oplus F \oplus G$ with $\operatorname{dim}(E)=k$ 
and $\operatorname{dim}(F)=1$, then there is a diffeomorphism $g$ arbitrarily $C^{1}$-close to $f$ having a homoclinic tangency associated to a saddle of s-index $k$. Therefore, since $f \in \mathrm{FT}^{1}(M)$, the homoclinic class $H(f)$ has a dominated splitting of the form $E \oplus F \oplus G$, where $\operatorname{dim}(E)=k$ and $F$ is one-dimensional. On the other hand, by [2], as $f$ is a $C^{1}$-generic diffeomorphism the homoclinic class $H(f)$ contains saddles of s-indices $k, k+1 \in[i, j]$. Now we can apply Theorem 1 to get Corollary 2 ,

We close this introduction discussing the special case of robustly non-hyperbolic and transitive diffeomorphisms. We point out that the proof of our results makes a systematic use of the machinery recently developed about the dynamics and generic properties of homoclinic classes of $C^{1}$ diffeomorphisms, see Section 3 for details. Therefore our results are formulated for $C^{1}$-generic diffeomorphisms.

On the other hand, the setting of robustly transitive and non-hyperbolic diffeomorphisms is a natural framework for considering Question 1 for open sets of non-hyperbolic diffeomorphisms. Moreover, in a recent paper, Nalsky gave examples of open sets of diffeomorphisms exhibiting non-hyperbolic ergodic measures supported on the whole homoclinic classes, [28]. In his case, one essentially has a skew product over a hyperbolic diffeomorphism in a base and a circle as a fiber. This example improves the construction in [21] mentioned before. Motivated by these results we want to formulate the partial case of the Conjecture 1 that looks more approachable:

Conjecture 2. Let $M$ be a closed manifold with $\operatorname{dim}(M) \geq 3$. Consider an open subset $\mathcal{T}(M)$ of $\operatorname{Diff}^{1}(M)$ consisting of non-hyperbolic transitive diffemorphisms $f$ such that there is a $D f$-invariant dominated splitting $T M=E \oplus F \oplus G$, where $F$ has dimension one and is non-hyperbolic. Then there is an open and dense subset $\mathcal{N}(M)$ of $\mathcal{T}(M)$ consisting of diffeomorphisms $f$ having a non-hyperbolic ergodic measure $\mu_{f}$ with full support, $\operatorname{supp} \mu_{f}=M$.

Notice that the approach used in this paper cannot be used straightforwardly to settle this conjecture. Indeed, our construction involves measures supported on periodic orbits, and therefore periodic orbits are dense in the support of the measure that we obtain. It is an open question whether periodic orbits are dense in a nonwandering set for an open and dense subset of the set of robustly transitive diffeomorphisms; $C^{1}$-Closing Lemma [32] implies this property only for a residual set of $C^{1}$-diffeomorphisms. 
This paper is organized as follows. In Section 2 we develop the idea initially suggested by Ilyashenko in [21] for constructing ergodic invariant measures as limit of measures supported on periodic orbits. In Section 3 we state properties of homoclinic classes of $C^{1}$-generic diffeomorphisms and recall results about heterodimensional cycles. Finally, in Section 4 we construct a collection of atomic measures supported on periodic orbits whose limit is a non-hyperbolic ergodic measure with full support, thus proving our main result.

\section{Acknowledgments}

The authors thank the financial support and hospitality of ICTP. We also express our gratitude to the organizers of the conferences "School and Workshop on Dynamical Systems", Trieste (2008), for creating a productive atmosphere for developing this paper. Also we are grateful to the organizers of the "International Workshop on Global Dynamics Beyond Uniform Hyperbolicity", Beijing (2009), where this paper was completed. L.J.D. thanks the financial support and warm hospitality of UC Irvine during his stay for preparing this paper. Ch.B. was partially supported by ANR project "DynNonHyp" BLAN082-313375, L.J.D. by CNPq, Faperj (Cientista do Nosso Estado and Infraestrutura), and PRONEX, and A.G. by grant NSF DMS-0901627.

\section{Non-hyperbolic ergodic measures as limits of atomic measures}

The approach suggested in [21] allows to construct non-hyperbolic ergodic invariant measures as limits of measures supported on special sequences of periodic points. Here we show that the support of the limit measure constructed in this way is a "topological limit" of the sequence of periodic points, see Proposition 2.7 for details.

\subsection{Ergodicity, invariant direction fields, and Lyapunov exponents}

Consider a diffeomorphism $f: M \rightarrow M$ and a closed invariant set $\Delta \subset M$. Assume that there is a $D f$-invariant continuous direction field $E=\left(E_{x}\right)_{x \in \Delta}$ in $\Delta$. Then for every invariant measure $\mu$ whose support is contained in $\Delta$ one of 
the Lyapunov exponents of $\mu$ is associated to $E$ (denote it by $\chi^{E}$ ). Namely, for $\mu$-a.e. $x \in M$ and for every non-zero vector $v \in E_{x} \subset T_{x} M$,

$$
\lim _{n \rightarrow \infty} \frac{1}{n} \log \left|D f^{n}(v)\right|=\chi^{E}(\mu) .
$$

In this section, by convergence of a sequence of measures we mean $*$-weak convergence: $\mu_{n}$ converges to $\mu$, if for any continuous function $\varphi: M \rightarrow \mathbb{R}$ it holds

$$
\int \varphi d \mu_{n} \rightarrow \int \varphi d \mu, \quad \text { as } n \rightarrow \infty .
$$

To obtain non-hyperbolic measures we will use the following statement.

Lemma 2.1 ([15], Lemma 2.1, or [21], Lemma 1). Let diffeomorphism $f: M \rightarrow$ $M$ have an invariant continuous direction field $E$ in an $f$-invariant closed set $\Delta \subset M$. Let $\mu_{n}$ and $\mu$ be ergodic probability measures with supports in $\Delta$, and $\mu_{n} \rightarrow \mu$ as $n \rightarrow \infty$. Then $\chi^{E}\left(\mu_{n}\right) \rightarrow \chi^{E}(\mu)$.

\subsection{Sufficient conditions for ergodicity and convergence}

In this section we state an improved version of Lemma 2 from [21]. We need the following definition.

Definition 2.2 (n-measure). Let $\mathfrak{G}$ be a continuous map of a metric compact space $\mathfrak{Q}$ into itself. A $n$-measure of the point $x_{0}$ is an atomic measure uniformly distributed on $n$ subsequent iterations of the point $x_{0}$ under the map $\mathfrak{G}$ :

$$
\nu_{n}\left(x_{0}\right)=\frac{1}{n} \sum_{i=0}^{n-1} \delta_{\mathfrak{G}^{i}\left(x_{0}\right)},
$$

where $\delta_{x}$ is $\delta$-measure supported at point $x$.

Lemma 2.3. Let $\left\{X_{n}\right\}$ be a sequence of periodic orbits with increasing periods $\pi\left(X_{n}\right)$ of a continuous map $\mathfrak{G}$ of a compact metric space $\mathfrak{Q}$ into itself. For each $n$, let $\mu_{n}$ be the probability atomic measure uniformly distributed on the orbit $X_{n}$.

Assume that for every $\varepsilon>0$ and every $m \in \mathbb{N}$ there exits a subset $\widetilde{X}_{m, \varepsilon} \subset X_{m}$ such that the following holds. For each continuous function $\varphi$ on $\mathfrak{Q}$ there exists $N=N(\varepsilon, \varphi) \in \mathbb{N}$ such that for all $m>N$ the following conditions are satisfied: 
1. $\mu_{m}\left(\widetilde{X}_{m, \varepsilon}\right)=\frac{\# \widetilde{X}_{m, \varepsilon}}{\# X_{m}}>1-\varepsilon(\# X$ the cardinality of the finite set $X)$ and

2. for any $n$, such that $m>n \geq N$, and for all $x \in \widetilde{X}_{m, \varepsilon}$ it holds

$$
\left|\int \varphi d \nu_{\pi\left(X_{n}\right)}(x)-\int \varphi d \mu_{n}\right|<\varepsilon
$$

Then the sequence of atomic measures $\left\{\mu_{n}\right\}$ has a limit, and the limit measure is ergodic.

Proof. Lemma 2 from [21] claims that under conditions of Lemma 2.3 every limit point $\mu$ of the sequence $\left\{\mu_{n}\right\}$ is an ergodic measure. Therefore we just need to show that the sequence of measures $\left\{\mu_{n}\right\}$ has a $*$-weak limit. In order to do that it is enough to show that for any continuous function $\varphi$ on $\mathfrak{Q}$ the sequence $\left\{\int \varphi d \mu_{n}\right\}$ is Cauchy and thus converges. Indeed, since $\left\{\mu_{n}\right\}$ has a convergent subsequence, $\mu_{n_{k}} \rightarrow \mu$ as $k \rightarrow \infty$, this implies that $\int \varphi d \mu_{n} \rightarrow \int \varphi d \mu$ and therefore $\mu_{n} \rightarrow \mu$ as $n \rightarrow \infty$.

Fix a continuous function $\varphi$ on $\mathfrak{Q}$. It is enough to show that the sequence $\left\{\int \varphi d \mu_{n}\right\}$ is Cauchy. Since $\mathfrak{Q}$ is compact, $\varphi$ is bounded, $|\varphi|<M$ for some $M>0$. Fix small $\varepsilon>0$ and consider the sets $\widetilde{X}_{m, \varepsilon} \subset X_{m}$ such that for all $m>$ $N=N(\varepsilon, \varphi)$ properties (1) and (2) hold. Now, for a given $m>n>N(\varepsilon, \varphi)$, we have

$$
\begin{aligned}
\sum_{x \in X_{m}} \int \varphi d \nu_{\pi\left(X_{n}\right)}(x) & =\sum_{x \in X_{m}}\left(\frac{1}{\pi\left(X_{n}\right)} \sum_{i=0}^{\pi\left(X_{n}\right)-1} \varphi\left(\mathfrak{G}^{i}(x)\right)\right)= \\
& =\frac{1}{\pi\left(X_{n}\right)} \sum_{x \in X_{m}} \sum_{i=0}^{\pi\left(X_{n}\right)-1} \varphi\left(\mathfrak{G}^{i}(x)\right)=\frac{1}{\pi\left(X_{n}\right)} \sum_{x \in X_{m}} \pi\left(X_{n}\right) \varphi(x)= \\
& =\sum_{x \in X_{m}} \varphi(x)=\pi\left(X_{m}\right) \int \varphi d \mu_{m} .
\end{aligned}
$$


Therefore we can estimate

$$
\begin{aligned}
\left|\int \varphi d \mu_{m}-\int \varphi d \mu_{n}\right|= & \frac{1}{\pi\left(X_{m}\right)}\left|\pi\left(X_{m}\right) \int \varphi d \mu_{m}-\pi\left(X_{m}\right) \int \varphi d \mu_{n}\right|= \\
= & \frac{1}{\pi\left(X_{m}\right)}\left|\sum_{x \in X_{m}} \int \varphi d \nu_{\pi\left(X_{n}\right)}(x)-\pi\left(X_{m}\right) \int \varphi d \mu_{n}\right| \leq \\
\leq & \frac{1}{\pi\left(X_{m}\right)}\left|\sum_{x \in \tilde{X}_{m, \varepsilon}}\left(\int \varphi d \nu_{\pi\left(X_{n}\right)}(x)-\int \varphi d \mu_{n}\right)\right|+ \\
& +\frac{1}{\pi\left(X_{m}\right)}\left|\sum_{x \notin \tilde{X}_{m, \varepsilon}}\left(\int \varphi d \nu_{\pi\left(X_{n}\right)}(x)-\int \varphi d \mu_{n}\right)\right| .
\end{aligned}
$$

Finally, from condition (2) in the lemma, $|\varphi|<M$, and condition (1), we have

$$
\left|\int \varphi d \mu_{m}-\int \varphi d \mu_{n}\right| \leq \frac{1}{\pi\left(X_{m}\right)}\left(\pi\left(X_{m}\right) \varepsilon+2 M \pi\left(X_{m}\right) \varepsilon\right)=(1+2 M) \varepsilon .
$$

So the sequence $\left\{\int \varphi d \mu_{n}\right\}$ is Cauchy, and Lemma 2.3 is proved.

\subsection{Sufficient conditions for existence of an invariant non- hyperbolic measure with large support}

In this section, we state an improved version of Proposition 2.5 from [15], where we add a description of the support of the resulting limit measure. We need the following definition.

Definition 2.4 (Good approximations). A periodic orbit $Y$ of a map $\mathfrak{G}$ of a compact metric space $\mathfrak{Q}$ into itself is a $(\gamma, \varkappa)$-good approximation of the periodic orbit $X$ of $\mathfrak{G}$ if the following holds.

- There exists a subset $\Gamma$ of $Y$ and a projection $\rho: \Gamma \rightarrow X$ such that

$$
\operatorname{dist}\left(\mathfrak{G}^{j}(y), \mathfrak{G}^{j}(\rho(y))\right)<\gamma,
$$

for every $y \in \Gamma$ and every $j=0,1, \ldots, \pi(X)-1$; 
- $\frac{\# \Gamma}{\# Y} \geq \varkappa$;

- $\# \rho^{-1}(x)$ is the same for all $x \in X$.

Lemma 2.5. Let $\left\{X_{n}\right\}$ be a sequence of periodic orbits with increasing periods $\pi\left(X_{n}\right)$ of a continuous map $\mathfrak{G}$ of a compact metric space $\mathfrak{Q}$ into itself. For each $n$, let $\mu_{n}$ be the probability atomic measure uniformly distributed on the orbit $X_{n}$.

Assume that there are sequences of numbers $\left\{\gamma_{n}\right\}_{n \in \mathbb{N}}, \gamma_{n}>0$, and $\left\{\varkappa_{n}\right\}_{n \in \mathbb{N}}$, $\varkappa_{n} \in(0,1]$, such that

1. for each $n \in \mathbb{N}$ the orbit $X_{n+1}$ is a $\left(\gamma_{n}, \varkappa_{n}\right)$-good approximation of $X_{n}$;

2. $\sum_{n=1}^{\infty} \gamma_{n}<\infty$;

3. $\prod_{n=1}^{\infty} \varkappa_{n} \in(0,1]$.

Then the sequence of atomic measures $\left\{\mu_{n}\right\}$ has a limit $\mu$. The limit measure $\mu$ is ergodic, and

$$
\operatorname{supp} \mu=\bigcap_{k=1}^{\infty}\left(\overline{\bigcup_{l=k}^{\infty} X_{l}}\right) \equiv \mathbf{X} \text {. }
$$

In other words, supp $\mu$ is a topological limit of the sequence of orbits $X_{n}$,

$$
\operatorname{supp} \mu=\left\{y \in \mathfrak{Q} \mid \exists m_{i} \rightarrow \infty \text { and } x_{i} \in X_{m_{i}} \text { such that } \lim _{i \rightarrow \infty} x_{i}=y\right\} .
$$

Proof. Let us check first that the conditions of the Lemma 2.3 are satisfied by the sequence of orbits $X_{n}$.

Take arbitrary $\varepsilon>0$ and continuous map $\varphi: \mathfrak{Q} \rightarrow \mathbb{R}$. By assumption 1 ), for orbits $\left\{X_{n}\right\}$ a sequence of subsets $\tilde{X}_{n} \subset X_{n}$ and a sequence of projections $\rho_{n}: \widetilde{X}_{n+1} \rightarrow X_{n}$ are defined such that:

$$
\prod_{n=1}^{\infty} \frac{\# \widetilde{X}_{n+1}}{\# X_{n+1}} \geq \prod_{n=1}^{\infty} \varkappa_{n}>0 .
$$

Choose $\delta=\delta(\varepsilon, \varphi)$ such that:

$$
\omega_{\delta}(\varphi):=\sup _{\operatorname{dist}(x, y)<\delta}|\varphi(x)-\varphi(y)|<\varepsilon .
$$


By assumption 2), we have $\sum_{n=1}^{\infty} \gamma_{n}<\infty$. Choose $N=N(\varepsilon, \varphi)$ such that the following holds:

$$
\sum_{N}^{\infty} \gamma_{k}<\delta(\varepsilon, \varphi) \quad \text { and } \quad \prod_{N}^{\infty} \varkappa_{k}>1-\varepsilon .
$$

Since the number of points in a pre-image for projections $\rho_{n}$ does not depend on a point in the image, a set $\widetilde{X}_{m, \varepsilon} \subset X_{m}$ where the total projection

$$
\rho_{m, N}=\rho_{m-1} \circ \cdots \circ \rho_{N}: \tilde{X}_{m, \varepsilon} \rightarrow X_{N}
$$

is defined contains most of the orbit $X_{m}$ :

$$
\frac{\# \tilde{X}_{m, \varepsilon}}{\# X_{m}} \geq \prod_{k=N}^{m-1} \varkappa_{k} \geq \prod_{N}^{\infty} \varkappa_{k}>1-\varepsilon .
$$

This implies 1) in Lemma 2.3.

Take arbitrary $m$ and $n$ with $m>n>N(\varepsilon, \varphi)$. By construction, on the set $\widetilde{X}_{m, \varepsilon}$ the total projection $\rho_{m, n}=\rho_{m-1} \circ \cdots \circ \rho_{n}$ is defined and for every point $x$ from the set $\tilde{X}_{m, \varepsilon} \subset X_{m}$ from the first part of equation (2) we have

$$
\operatorname{dist}\left(\mathfrak{G}^{j}(x), \mathfrak{G}^{j}\left(\rho_{m, n}(x)\right)\right)<\delta(\varepsilon, \varphi), \quad \text { for all } j=0,1, \ldots, \pi\left(X_{n}\right)-1 .
$$

Hence for $x \in \widetilde{X}_{m, \varepsilon}$ we have:

$$
\left|\int \varphi d \nu_{\pi\left(X_{n}\right)}(x)-\int \varphi d \mu_{n}\right|<\omega_{\delta}(\varphi)<\varepsilon .
$$

Thus all conditions of Lemma 2.3 are verified. Therefore the sequence $\left\{\mu_{n}\right\}$ has a limit $\mu$, and the limit measure $\mu$ is ergodic.

Now we need the following lemma. Denote by $U_{\delta}(x)$ the ball of radius $\delta$ centered at $x$.

Lemma 2.6. Set $r_{n}=\sum_{k=n}^{\infty} \gamma_{k}$. For every point $x \in X_{n}$ one has $\mu\left(\overline{U_{r_{n}}(x)}\right)>0$.

Proof. Take any point $x \in X_{n}$. Notice that in its $\gamma_{n}$-neighborhood there are at least $\frac{\# \widetilde{X}_{n+1, \varepsilon}}{\pi\left(X_{n}\right)}$ points of the orbit of $X_{n+1}$, where

$$
\frac{\# \widetilde{X}_{n+1, \varepsilon}}{\pi\left(X_{n}\right)}=\frac{\# \tilde{X}_{n+1, \varepsilon}}{\pi\left(X_{n+1}\right)} \frac{\pi\left(X_{n+1}\right)}{\pi\left(X_{n}\right)} \geq \varkappa_{n} \frac{\pi\left(X_{n+1}\right)}{\pi\left(X_{n}\right)} \equiv \bar{\varkappa}_{n} .
$$


Therefore,

$$
\mu_{n+1}\left(U_{\gamma_{n}}(x)\right) \geq \frac{\varkappa_{n} \frac{\pi\left(X_{n+1}\right)}{\pi\left(X_{n}\right)}}{\pi\left(X_{n+1}\right)}=\varkappa_{n} \frac{1}{\pi\left(X_{n}\right)}=\varkappa_{n} \mu_{n}(\{x\}) .
$$

In the neighborhood $U_{\gamma_{n}}(x)$ there are $p$ different points $x_{1}, \ldots, x_{p}$ of the orbit of $X_{n+1}$, where $p \geq \bar{\varkappa}_{n}$. Notice that, by equation (44), for each of these points $x_{i}$ the pre-image $\rho_{n+2}^{-1}\left(x_{i}\right)$ consists of at least

$$
\frac{\# \tilde{X}_{n+2, \varepsilon}}{\pi\left(X_{n+1}\right)} \geq \varkappa_{n+1} \frac{\pi\left(X_{n+2}\right)}{\pi\left(X_{n+1}\right)}
$$

points. The sets $\rho_{n+2}^{-1}\left(x_{i}\right)$ and $\rho_{n+2}^{-1}\left(x_{j}\right)$ are disjoint for $i \neq j$. Therefore we have

$$
\begin{aligned}
\mu_{n+2}\left(U_{\gamma_{n+1}+\gamma_{n}}(x)\right) & \geq\left[p \varkappa_{n+1} \frac{\pi\left(X_{n+2}\right)}{\pi\left(X_{n+1}\right)}\right] \frac{1}{\pi\left(X_{n+2}\right)} \geq \\
& \geq\left(\varkappa_{n} \frac{\pi\left(X_{n+1}\right)}{\pi\left(X_{n}\right)}\right) \varkappa_{n+1} \frac{1}{\pi\left(X_{n+1}\right)}=\frac{\varkappa_{n} \varkappa_{n+1}}{\pi\left(X_{n}\right)}= \\
& =\varkappa_{n} \varkappa_{n+1} \mu_{n}(\{x\}) .
\end{aligned}
$$

Thus arguing inductively we have, for every $x \in X_{n}$,

$$
\mu_{n+\ell}\left(U_{\gamma_{n+\ell}+\cdots+\gamma_{n+1}+\gamma_{n}}(x)\right) \geq\left(\varkappa_{n+\ell} \cdots \varkappa_{n+1} \varkappa_{n}\right) \mu_{n}(\{x\}) .
$$

Taking a limit and taking into account the condition 3 in Lemma 2.5, we have:

$$
\mu\left(\overline{U_{r_{n}}(x)}\right) \geq\left(\prod_{k=n}^{\infty} \varkappa_{k}\right) \mu_{n}(\{x\})>0, \quad \text { where } r_{n}=\sum_{k=n}^{\infty} \gamma_{k} .
$$

Therefore, Lemma 2.6 holds.

Now let us show that the support of $\mu$ is the topological limit $\mathbf{X}$ of the sequence of orbits $X_{n}$. It is a general fact that for any convergent sequence of measures the support of the limit is a subset of the topological limit of the sequence of supports, therefore supp $\mu \subseteq \mathbf{X}$. We need to show that in our case we also have $\mathbf{X} \subseteq \operatorname{supp} \mu$. 
Take any point $z \in \mathbf{X}$. It is enough to show that arbitrary small $\varepsilon>0$ one has $\mu\left(U_{\varepsilon}(z)\right)>0$. Choose $n^{\prime} \in \mathbb{N}$ large enough to guarantee that

$$
X_{n^{\prime}} \cap U_{\varepsilon / 2}(z) \neq \emptyset \quad \text { and } \quad r_{n^{\prime}}<\varepsilon / 2 .
$$

Take any $z^{\prime} \in X_{n^{\prime}} \cap U_{\varepsilon / 2}(z)$. We have $U_{\varepsilon}(z) \supset \overline{U_{r_{n^{\prime}}}\left(z^{\prime}\right)}$, and due to Lemma 2.6 $\mu\left(\overline{U_{r_{n^{\prime}}}\left(z^{\prime}\right)}\right)>0$. Hence $\mu\left(U_{\varepsilon}(z)\right)>0$, and therefore $z \in \operatorname{supp} \mu$.

This completes the proof of Lemma 2.5.

Proposition 2.7. Assume that a diffeomorphism $f: M \rightarrow M$ has the following properties:

1) there exists an $f$-invariant closed set $\Delta \subset M$ such that $f$ has an invariant continuous direction field $E$ in $\Delta$;

2) there exists a sequence of periodic orbits $\left\{X_{n}\right\}_{n=1}^{\infty}, X_{n} \subset \Delta$, of $f$ whose periods $\pi\left(X_{n}\right)$ tend to infinity as $n \rightarrow \infty$.

Denote by $\chi^{E}(X)$ the Lyapunov exponent along the periodic orbit $X$ with respect to the invariant direction field $E$.

3) There exists a sequence of numbers $\left\{\gamma_{n}\right\}_{n=1}^{\infty}, \gamma_{n}>0$, and a constant $C>0$ such that for each $n$ the orbit $X_{n+1}$ is a $\left(\gamma_{n}, 1-C\left|\chi^{E}\left(X_{n}\right)\right|\right)$-good approximation of the orbit $X_{n}$;

4) let $d_{n}$ be the minimal distance between the points of the orbit $X_{n}$, then

$$
\gamma_{n}<\frac{\min _{1 \leq i \leq n} d_{i}}{3 \cdot 2^{n}}
$$

5) there exits a constant $\xi \in(0,1)$ such that for every $n$

$$
\left|\chi^{E}\left(X_{n+1}\right)\right|<\xi\left|\chi^{E}\left(X_{n}\right)\right| \text {. }
$$

Then the sequence of atomic measures $\mu_{n}$ supported on periodic orbits $X_{n}$ has a (*-weak) limit $\mu$. The measure $\mu$ is a non-hyperbolic ergodic invariant measure of $f$, and supp $\mu$ is uncountable. Moreover, supp $\mu$ is a topological limit of the sequence of orbits $X_{n}$, i.e.

$$
\operatorname{supp} \mu=\bigcap_{k=1}^{\infty}\left(\overline{\bigcup_{l=k}^{\infty} X_{l}}\right) \text {. }
$$


Proof. Assumptions 1)-5) of Proposition 2.7 imply that conditions of Lemma 2.5 are satisfied. Therefore there exists a $\operatorname{limit}_{n \rightarrow \infty} \mu_{n}=\mu$, the measure $\mu$ is an ergodic invariant measure of $f$, and supp $\mu$ is a topological limit of the sequence of orbits $X_{n}$. Due to Lemma 2.1 and property 5), measure $\mu$ is non-hyperbolic. Finally, Proposition 2.5 from [15] implies that supp $\mu$ is uncountable.

\section{Homoclinic classes: $C^{1}$-generic properties and generation of cycles}

In this section we recall some known results on homoclinic classes and heterodimensional cycles that will be used later in our construction.

\subsection{Generic properties}

Here we state properties of homoclinic classes of $C^{1}$-generic diffeomorphisms. There is a residual subset $\mathcal{G}$ of $\operatorname{Diff}^{1}(M)$ such that every diffeomorphism $f \in \mathcal{G}$ satisfies properties $\mathbf{R} \mathbf{1})-\mathbf{R} 5)$ below.

R1) Every homoclinic class of $f \in \mathcal{G}$ depends continuously on $f$ in Hausdorff metric, see [13]. Moreover, if the homoclinic class contains saddles of indices $a$ and $b, a<b$, it also contains saddles of index $c$, for every $c \in(a, b) \cap \mathbb{N}$. See [2, Theorem 1].

Consider a hyperbolic periodic point $P_{f}$ of a diffeomorphism $f$. It is well known that there are open neighborhoods $U$ of $P_{f}$ in the manifold and $\mathcal{U}$ of $f$ in Diff ${ }^{1}(M)$ such that every $g \in \mathcal{U}$ has a unique hyperbolic periodic point $P_{g}$ of the same period as $P_{f}$ in $U$. The point $P_{g}$ is called the continuation of $P_{f}$.

Recall that two saddles $P_{f}$ and $Q_{f}$ are homoclinically related if their invariant manifolds $W^{\mathrm{s}}\left(P_{f}\right)$ and $W^{\mathrm{u}}\left(Q_{f}\right)$ and $W^{\mathrm{u}}\left(P_{f}\right)$ and $W^{\mathrm{s}}\left(Q_{f}\right)$ have non-empty transverse intersections. In this case, the homoclinic classes of $P_{f}$ and of $Q_{f}$ coincide and the saddles have the same index. Moreover, the continuations $P_{g}$ and $Q_{g}$ are also homoclinically related for all $g$ close to $f$.

Definition 3.1 (Persistently linked saddles). Consider a pair of hyperbolic saddles $P_{f}$ and $Q_{f}$ whose continuations are defined for every $f$ in an open set $\mathcal{U}$ of $\operatorname{Diff}^{1}(M)$. The saddles $P_{f}$ and $Q_{f}$ are persistently linked in $\mathcal{U}$ if there is a residual subset $\mathcal{R}$ of $\mathcal{U}$ such that $H\left(P_{f}, f\right)=H\left(Q_{f}, f\right)$ for all $f \in \mathcal{U} \cap \mathcal{R}$. 
R2) Given any $f \in \mathcal{G}$ and any pair of saddles $P_{f}$ and $Q_{f}$ of $f$, there is a neighborhood $\mathcal{U}_{f}$ of $f$ such that either $P_{f}$ and $Q_{f}$ are persistently linked in $\mathcal{U}_{f}$ or $H\left(P_{g}, g\right) \cap H\left(Q_{g}, g\right)=\emptyset$ for all $g \in \mathcal{U}_{f} \cap \mathcal{G}$. Moreover, If the saddles $P_{f}$ and $Q_{f}$ have the same index they are homoclinically related. See [2, Lemma 2.1].

Definition 3.2 (Saddle with real multipliers). Let $P$ be a periodic point of period $\pi(P)$ of a diffeomorphism $f$. We say that $P$ has real multipliers if every eigenvalue $\lambda$ of $D f^{\pi(P)}(P)$ is real and has multiplicity one, and two different eigenvalues of $D f^{\pi(P)}(P)$ have different absolute values. We order the eigenvalues of $D f^{\pi(P)}(P)$ in increasing ordering according their absolute values $\left|\lambda^{1}(P)\right|<\cdots<\left|\lambda^{\mathbf{m}}(P)\right|$ and say that $\lambda^{k}(P)$ is the $k$-th multiplier of $P$.

Definition 3.3 (s- and u-index). Let $P$ be a hyperbolic saddle. By s-index of $P$ we mean the number of multipliers of $P$ with absolute value less than one, and by $\mathrm{u}$-index the number of multipliers of $P$ with absolute value greater than one.

Let $P$ be a saddle with real multipliers, and suppose that s-index $(P)=t+1$. Consider the bundle $E^{\mathrm{ss}} \subset T_{P} M$ corresponding to the first $t$ contracting eigenvalues of $P$ and the strong stable manifold $W^{\text {ss }}(P)$ of $P$ (the only $f$-invariant manifold of dimension $t$ tangent to the strong stable direction $\left.E^{\mathrm{ss}}\right)$.

Definition 3.4 (s- and u-biaccumulation). A hyperbolic saddle $P$ with real multipliers is s-biaccumulated (by its homoclinic points) if both connected components of $W_{\mathrm{loc}}^{\mathrm{s}}(P) \backslash W_{\mathrm{loc}}^{\mathrm{ss}}(P)$ contain transverse homoclinic points of $P$. Define also u-biaccumulation by homoclinic points in a similar way.

Note that s- and u-biaccumulation are open properties.

Given a saddle $P$, we denote by $\operatorname{Per}_{\mathbb{R}}(H(P, f))$ the saddles homoclinically related to $P$ having real multipliers. Clearly, $\operatorname{Per}_{\mathbb{R}}(H(P, f)) \subset H(P, f)$.

R3) For every diffeomorphism $f \in \mathcal{G}$ and every saddle $P$ of $f$ whose homoclinic class is non-trivial the set $\operatorname{Per}_{\mathbb{R}}(H(P, f))$ is dense in the whole homoclinic class $H(P, f)$. Moreover, the saddles from $\operatorname{Per}_{\mathbb{R}}(H(P, f))$ which are s- (or u-) biaccumulated are also dense in $H(P, f)$. See [2, Proposition 2.3] and [15, Lemma 3.4].

R4) Consider $f \in \mathcal{G}$ and a saddle $P$ of $f$ whose homoclinic class is non-trivial. For every $\epsilon>0$ and every $k=1, \ldots, \mathbf{m}$, one has that

$$
\overline{\left(\left\{S \in \operatorname{Per}_{\mathbb{R}}(H(P, f)):\left|\chi^{k}(S)-\chi^{k}(P)\right|<\epsilon\right\}\right)}=H(P, f) .
$$


Assume now that $\chi^{k}(P)<0$ and suppose that $H(P, f)$ contains a saddle $Q$ with $\chi^{k}(Q)>0$. Then one has that

$$
\overline{\left(\left\{S \in \operatorname{Per}_{\mathbb{R}}(H(P, f)): \chi^{k}(S) \in(-\epsilon, 0)\right\}\right)}=H(P, f) .
$$

Moreover, we can additionally assume that the dense subsets of $H(P, f)$ above consist of saddles with the biaccumulation properties. For details see [2, 7, 15].

R5) We will actually need a slightly stronger property.

Lemma 3.5. Consider any $f \in \mathcal{G}$ and any hyperbolic saddle $P_{f}$ whose homoclinic class is non-trivial. For every $\epsilon>0$ and $k=1, \ldots, \mathbf{m}$, the homoclinic class $H\left(P_{f}, f\right)$ of $f$ contains a saddle $Y_{f} \in H\left(P_{f}, f\right)$ with real multipliers such that $Y_{f}$ is homoclinically related to $P_{f}$, its orbit is $\epsilon$-dense in the homoclinic class $H\left(P_{f}, f\right)$, and the Lyapunov exponent $\chi^{k}\left(Y_{f}\right)$ is $\epsilon$-close to $\chi^{k}\left(P_{f}\right)$.

Proof. Indeed, for a given $\varepsilon>0$, by $\mathbf{R} 4$ ) there exists a finite collection of periodic saddles $\left\{P_{i}\right\}_{i=1,2, \ldots, m}$ which is $\varepsilon / 2$-dense in $H\left(P_{f}, f\right)$, and their Lyapunov exponents $\chi^{k}\left(P_{i}\right)$ are $\varepsilon$-close to $\chi^{k}\left(P_{f}\right)$. Moreover, as $f \in \mathcal{G}$, by R2) these saddles are homoclinically related. This implies that there exists a locally maximal transitive hyperbolic set $\Lambda_{f}$ with $P_{i} \in \Lambda_{f}$, for all $i=1, \ldots, m$. Then there is a periodic saddle $Y_{f} \in \Lambda_{f}$ that spends an arbitrary large portion of time in an arbitrary small neighborhood of the initial collection of saddles, and is $\varepsilon / 2$-dense in $\Lambda_{f}$. This implies that the orbit of $Y_{f}$ is $\varepsilon$-dense in $H\left(P_{f}, f\right)$ and its Lyapunov exponent $\chi^{k}\left(Y_{f}\right)$ is close to the Lyapunov exponent $\chi^{k}\left(P_{f}\right)$, completing the proof of the lemma.

\subsection{Heterodimensional cycles}

In this section, we state results that allow us to generate heterodimesional cycles for saddles in a non-hyperbolic homoclinic classes. Roughly speaking, we need to be able to create a cycle associated with a given pair of homoclinically linked saddles of different indices (Proposition 3.6); to produce a locally dense set of diffeomorphisms with these type of cycles (Proposition 3.7); and, finally, to use these cycles to generate periodic saddles with some special properties 
(Proposition 3.8) that will allow to use Proposition 2.7 later to obtain nonhyperbolic ergodic measures with large support.

The following result, which is a consequence of the Connecting Lemma in [23], can be found in [15, Proposition 3.5]. Recall that two saddles $P$ and $Q$ of different indices have a heterodimensional cycle if its invariant manifolds meet cyclically, $W^{\mathrm{s}}(P) \cap W^{\mathrm{u}}(Q) \neq \emptyset$ and $W^{\mathrm{u}}(P) \cap W^{\mathrm{s}}(Q) \neq \emptyset$. The cycle has coindex one if s-index $(P)=\operatorname{s-index} Q \pm 1$.

Proposition 3.6. Let $\mathcal{U}$ be an open set of $\operatorname{Diff}^{1}(M)$ such that there are saddles $P_{f}$ and $Q_{f}$ (depending continuously on $f \in \mathcal{U}$ ) with consecutive indices which are persistently linked in $\mathcal{U}$. Then there is a dense subset $\mathcal{H}$ of $\mathcal{U}$ such that every diffeomorphism $f \in \mathcal{H}$ has a coindex one heterodimensional cycle associated to saddles $A_{f} \in \operatorname{Per}_{\mathbb{R}}\left(H\left(P_{f}, f\right)\right)$ and $B_{f} \in \operatorname{Per}_{\mathbb{R}}\left(H\left(Q_{f}, f\right)\right)$.

The following statements are straightforward reformulations of Propositions 4.3 and 4.5 from [15] better suited for our case.

Proposition 3.7. Let $f$ have a cycle associated to saddles $A_{f}$ and $B_{f}$ such that

- $A_{f}$ and $B_{f}$ have real multipliers and s-index $\left(A_{f}\right)=\operatorname{s-index}\left(B_{f}\right)+1$;

- $A_{f}$ is s-biaccumulated and $B_{f}$ is u-biaccumulated;

Then arbitrarily $C^{1}$-close to $f$ there are an open set $\mathcal{E} \subset \operatorname{Diff}^{1}(M)$ and dense subset $\mathcal{D} \subset \mathcal{E}$ such that every $g \in \mathcal{D}$ has a cycle associated with $A_{g}$ and $B_{g}$.

Proposition 3.8. ([15, Proposition 4.5]) Let $f$ be a diffeomorphism with a heterodimensional cycle associated to saddles $A_{f}$ and $B_{f}$ such that

(i) the saddles $A_{f}$ and $B_{f}$ have real multipliers;

(ii) $\mathrm{s}-$ index $\left(A_{f}\right)=i+1$ and $\mathrm{s}-\operatorname{index}\left(B_{f}\right)=i$;

(iii) $A_{f}$ is s-biaccumulated, and $B_{f}$ is u-biaccumulated.

Fix neighborhoods $U_{B}$ of the orbit of $B_{f}$ and $U_{A}$ of the orbit of $A_{f}$. Then there are sequences of natural numbers $\ell_{k}, m_{k}$, that tend to infinity as $k \rightarrow \infty$, and a sequence of diffeomorphisms $f_{k}, f_{k} \rightarrow f$ as $k \rightarrow \infty$, such that $f_{k}$ coincides with $f$ along the orbits of $A_{f}$ and $B_{f}$, and has a hyperbolic saddle $R_{k}$ having real multipliers with the following properties: 
(1) the orbit of the saddle $R_{k}$ spends a fixed number $t_{(a, b)}$ (independent of $k$ ) of iterates to go from $U_{B}$ to $U_{A}$, then it remains $\ell_{k} \pi\left(A_{f}\right)$ iterates in $U_{A}$, then it takes a fixed number of iterates $t_{(b, a)}$ (independent of $k$ ) to go from $U_{A}$ to $U_{B}$, and finally it remains $m_{k} \pi\left(B_{f}\right)$ iterates in $U_{B}$. In particular, there is a constant $t \in \mathbb{N}$ independent of $k$ such that the period of $R_{k}$ is $\pi\left(R_{k}\right)=m_{k} \pi\left(B_{f}\right)+\ell_{k} \pi\left(A_{f}\right)+t ;$

(2) there is a constant $\Theta>0$ independent of $k$ such that the central multiplier of $R_{k}$ satisfies $\Theta^{-1}<\left|\lambda^{i+1}\left(R_{k}\right)\right|<\Theta$.

Suppose also that $\left|\lambda^{i+1}\left(A_{f}\right)\right| \in\left(\frac{1}{\sqrt{2}}, 1\right) 1$. Then

(3) $R_{k}$ has the same index as $B_{f}$ and is homoclinically related to $B_{f}$;

(4) $W^{\mathrm{s}}\left(R_{k}\right)$ intersects $W^{\mathrm{uu}}\left(R_{k}\right)$, and $W^{\mathrm{uu}}\left(R_{k}\right)$ intersects $W^{\mathrm{s}}\left(B_{f}\right)$. Moreover, these intersections are quasi-transverse;

(5) there is a heterodimensional cycle associated to $R_{k}$ and $A_{f}$.

Remark 3.9. Notice that the condition (1) implies that there exists a sequence $\delta_{k} \rightarrow 0^{+}$such that

$$
\mathcal{O}\left(A_{f}\right), \mathcal{O}\left(B_{f}\right) \subseteq B_{\delta_{k}}\left(\mathcal{O}\left(R_{k}\right)\right)
$$

Here $\mathcal{O}(X)$ denotes the full orbit of the point $X$.

Remark 3.10. In [15] we introduced $V$-related cycles in order to guarantee that all of the sets we consider are in the domain where a central direction field is defined. Here this property is one of the assumptions of the main result, so we do not need to focus on $V$-related cycles. Propositions 3.7 and 3.8 above are therefore weaker versions of Propositions 4.3 and 4.5 from [15].

\section{Main construction: tree of periodic points and limit measures}

In this section we use the results of previous sections to complete the proof of Theorem 1, Let $\mathcal{G}$ be the residual set of $\operatorname{Diff}^{1}(M)$ described in Section 3.1. The statement below is a local version of Theorem 1 .

\footnotetext{
${ }^{1}$ In [15] here just assumed that the $(i+1)$-th multiplier $\lambda^{i+1}\left(A_{f}\right)$ of $A_{f}$ is close to one. To be more accurate, here we replaced it by the condition that we actually needed for the proof, see equation (8) in [15].
} 
Theorem 2. Let $f \in \mathcal{G}$ have a homoclinic class $H(f)$ of $f$ such that

- $H(f)$ has a dominated splitting of the form $T_{H(f)} M=E \oplus F \oplus G$, where $F$ has dimension one,

- $H(f)=H\left(P_{f}, f\right)=H\left(Q_{f}, f\right)$, where the saddles $P_{f}$ and $Q_{f}$ have s-indices $\operatorname{dim}(E \oplus F)=\operatorname{dim}(E)+1$ and $\operatorname{dim}(E)$.

Then arbitrarily $C^{1}$-close to $f$ there exists a $C^{1}$-open set $\mathcal{Z} \subset \operatorname{Diff}^{1}(M)$ and a residual subset $\mathcal{R} \subset \mathcal{Z}$ such that every $g \in \mathcal{R}$ has a non-hyperbolic ergodic invariant measure whose support is the whole homoclinic class $H\left(P_{g}, g\right)$.

As in [15] (see also [2]), standard genericity arguments imply that Theorem 1 follows from Theorem 2 .

Before we continue, let us remind that existence of the dominated splitting is preserved by small perturbations.

Lemma 4.1 (Dominated splittings, Appendix B.1.1 in [10]). Assume that the homoclinic class $H\left(P_{f}, f\right)$ has a dominated splitting $T_{H\left(P_{f}, f\right)} M=E_{f} \oplus F_{f} \oplus G_{f}$. There are neighborhoods $V$ of $H\left(P_{f}, f\right)$ and $\mathcal{U}_{f}$ of $f$ such that for every $g \in \mathcal{U}_{f}$, the maximal invariant set $\Lambda_{g}(V)$ has a dominated splitting $T_{\Lambda_{g}(V)} M=E_{g} \oplus F_{g} \oplus$ $G_{g}$, where $\operatorname{dim} K_{f}=\operatorname{dim} K_{g}, K=E, F, G$. With a slight abuse of notation, we will omit the dependence on $g$ of this splitting.

\subsection{Construction of the sequences of periodic orbits}

Consider a homoclinic class $H(f)=H\left(P_{f}, f\right)=H\left(Q_{f}, f\right)$ of $f \in \mathcal{G}$ that satisfy the conditions of Theorem 2.

The genericity hypotheses $\mathbf{R 2}$ )-R3) imply that the homoclinic class $H(f)$ contains two saddles $A_{f}$ and $B_{f}$ with real multipliers such that

$$
\mathrm{s}-\operatorname{index}\left(A_{f}\right)=\mathrm{s}-\operatorname{index}\left(P_{f}\right), \quad \mathrm{s}-\operatorname{index}\left(B_{f}\right)=\mathrm{s}-\operatorname{index}\left(Q_{f}\right),
$$

and

$$
H(f)=H\left(A_{f}, f\right)=H\left(B_{f}, f\right)=H\left(P_{f}, f\right)=H\left(Q_{f}, f\right) .
$$

The homoclinic class $H(f)$ has a splitting $E \oplus F \oplus G$, where $F$ is one-dimensional. We can assume that the saddle $A_{f}$ is s-biaccumulated, the saddle $B_{f}$ is ubiaccumulated, and that the central multiplier of $A_{f}$ is close to one (it is enough to have $\left.\left|\lambda^{F}\left(A_{f}\right)\right| \in(0.9,1)\right)$, see generic conditions $\left.\left.\mathbf{R 3}\right)-\mathbf{R} 4\right)$. 
Recall that $\chi^{F}\left(A_{f}\right)$ denotes the central Lyapunov exponent of saddle $A_{f}$ corresponding to the central direction $F$. Fix a constant $C$ such that

$$
C>\frac{32}{\nu}, \text { where } \nu=\left|\chi^{F}\left(A_{f}, f\right)\right| .
$$

Lemma 4.2. Let $f$ satisfy the conditions of Theorem 0 and constants $C, \nu>0$ be as in (6). Given $\epsilon>0$, arbitrary close to $f$ there exists an open set $\mathcal{Z} \subset$ $\operatorname{Diff}^{1}(M)$ with the following properties.

1) For every $g \in \mathcal{Z} \cap \mathcal{G}$

$$
H(f) \subset B_{\epsilon / 2}\left(H\left(P_{g}, g\right)\right), \quad H\left(P_{g}, g\right) \subset B_{\epsilon / 2}(H(f)) .
$$

2) For every $g \in \mathcal{Z} \cap \mathcal{G}$, we have

- $H\left(P_{g}, g\right)=H\left(Q_{g}, g\right)=H\left(A_{g}, g\right)=H\left(B_{g}, g\right)$,

- there is a dominated splitting (with one-dimensional central direction) on $H(g)=H\left(P_{g}, g\right)$.

3) For each $g \in \mathcal{Z}$ there is a saddle $Y_{g} \subset H\left(P_{g}, g\right)$ with real multipliers such that the following holds.

- the orbit of $Y_{g}$ is $\epsilon$-dense in $H(g)$,

- $Y_{g}$ and $P_{g}$ are homoclinically related, hence $H\left(Y_{g}, g\right)=H\left(P_{g}, g\right)$,

- $\chi_{Y_{g}}^{F} \in[-2 \nu,-\nu / 2]$,

- the saddle $Y_{g}$ is s-biaccumulated.

4) There is a dense in $\mathcal{Z}$ countable subset $\mathcal{D} \subset \mathcal{Z}$ such that every $\phi \in \mathcal{D}$ has a cycle associated with the saddles $Y_{\phi}$ and $B_{\phi}$.

Proof. Since $f \in \mathcal{G}$, by property $\mathbf{R} 1$ ), continuous dependence of homoclinic classes of diffeomorphisms in $\mathcal{G}$, there is an open set $\mathcal{W} \subset \operatorname{Diff}^{1}(M), f \in \mathcal{W}$, such that for every $g \in \mathcal{W} \cap \mathcal{G}$ we have

$$
H(f) \subset B_{\epsilon / 2}\left(H\left(P_{g}, g\right)\right), \quad H\left(P_{g}, g\right) \subset B_{\epsilon / 2}(H(f)),
$$

obtaining property $\mathbf{1})$.

By Lemma 4.1 there is a dominated splitting (with one-dimensional central direction) on $H(g)=H\left(P_{g}, g\right)$ which is a continuation of $E \oplus F \oplus G$. In 
particular, such a splitting also has one dimensional central direction. Slightly abusing the notation, we will continue to denote this splitting by $E \oplus F \oplus G$. If $\mathcal{W}$ is small enough then for every $g \in \mathcal{W} \cap \mathcal{G}$, we have $H\left(P_{g}, g\right)=H\left(Q_{g}, g\right)=$ $H\left(A_{g}, g\right)=H\left(B_{g}, g\right)$, see $\left.\mathbf{R 2}\right)$. This gives $\left.\mathbf{2}\right)$.

Due to R5) there is $g$ arbitrary $C^{1}$ close to $f$ and a saddle $Y_{g} \subset H\left(P_{g}, g\right)$ with real multipliers such that its orbit is $\epsilon / 3$-dense in $H(g), H\left(Y_{g}, g\right)=H\left(P_{g}, g\right)$, $\chi_{Y_{g}}^{F} \in[-2 \nu,-\nu / 2]$, and which is s-biaccumulated, hence we have 3 ).

Finally, Proposition 3.6 implies that $C^{1}$-arbitrarily close to $g$ there is $h$ with a cycle corresponding to the saddles $Y_{h}$ and $B_{h}$. Therefore, by Proposition 3.7 , there is an open set $\mathcal{Z} \subset \mathcal{W}$ and a dense in $\mathcal{Z}$ countable subset $\mathcal{D} \subset \mathcal{Z}$ such that every $\phi \in \mathcal{D}$ has a cycle associated with the saddles $Y_{\phi}$ and $B_{\phi}$. This completes the proof of Lemma 4.2 .

Next proposition can be considered as an improved version of Proposition 5.3 from [15]. Notice that properties Z3) and Z4) allow to "spread" the constructed sequence of periodic orbits around the whole homoclinic class.

Proposition 4.3. Let $f$ satisfy the conditions of Theorem 2 and constants $C, \nu>0$ be as in (6). Fix a decreasing sequence $\left\{\epsilon_{N}\right\}_{N \in \mathbb{N}}$ of positive numbers, $\epsilon_{N} \rightarrow 0^{+}$as $N \rightarrow \infty$. Arbitrarily $C^{1}$-close to $f$ there exists a nested sequence of open sets

$$
\ldots \mathcal{Z}_{N} \subset \mathcal{Z}_{N-1} \subset \ldots \mathcal{Z}_{2} \subset \mathcal{Z}_{1} \subset \operatorname{Diff}^{1}(M)
$$

such that the following holds.

Z1) $A$ set $\mathcal{Z}_{N}$ is a dense open subset of $\mathcal{Z}_{N-1}$.

Z2) Every diffeomorphism $g \in \mathcal{Z}_{N}$ has a finite sequence of periodic saddles homoclinically related to $B_{g}$ (thus of the same index as $B_{g}$ )

$$
\left\{Q_{1, g}, Q_{2, g}, \ldots, Q_{N, g}\right\} \subset H\left(B_{g}, g\right)
$$

having real multipliers, satisfying the u-biaccumulation property, and of growing periods, $\pi\left(B_{g}\right)=\pi\left(Q_{1, g}\right)<\ldots<\pi\left(Q_{N, g}\right)$. Moreover, saddles $\left\{Q_{1, g}, Q_{2, g}, \ldots, Q_{N, g}\right\}$ depend continuously on $g$ when $g$ varies over $\mathcal{Z}_{N}$.

Z3) For any $N \in \mathbb{N}$ there exists a countable dense subset $\mathcal{D}_{N} \subset \mathcal{Z}_{N}$ such that every $g \in \mathcal{D}_{N}$ has a saddle $Y_{N, g}$ depending continuously on $g$ such that

- the g-orbit of $Y_{N, g}$ is $\epsilon_{N+1}$-dense in $H\left(B_{g}, g\right)$, 
- $\chi^{F}\left(Y_{N, g}\right) \in[-2 \nu,-\nu / 2]$,

- there is a heterodimensional cycle associated to the saddles $Y_{N, g}$ and $Q_{N, g}$.

Z4) There is a sequence of locally constant functions $\gamma_{N}: \mathcal{Z}_{N} \rightarrow(0,+\infty)$ such that for any $N \in \mathbb{N}$ and any $g \in \mathcal{Z}_{N+1}$ the orbit of $Q_{N+1, g}$ is a $\left(\gamma_{N}(g), 1-C\left|\chi^{F}\left(Q_{N, g}\right)\right|\right)$-good approximation of the orbit of $Q_{N, g}$ (recall Definition 2.4), where $C$ is the constant in (6). Moreover,

$$
B_{\epsilon_{N+1}}\left(\mathcal{O}\left(Q_{N+1, g}\right)\right) \supset \mathcal{O}\left(Y_{N, g}\right) .
$$

Z5) Take any $g \in \mathcal{Z}_{N}$. Let $d_{k}, 1 \leq k \leq N$, be the minimal distance between the points of the g-orbit of $Q_{k, g}$. Then

$$
\gamma_{N}(g)<\frac{\min _{1 \leq k \leq N} d_{k}}{3 \cdot 2^{N}} .
$$

Z6) For each $N \in \mathbb{N}$ and every $g \in \mathcal{Z}_{N+1}$

$$
\left|\chi^{F}\left(Q_{N+1, g}\right)\right|<\frac{1}{2}\left|\chi^{F}\left(Q_{N, g}\right)\right| .
$$

Proof. We prove Proposition 4.3 by induction.

As a set $\mathcal{Z}_{1}$ we take the set $\mathcal{Z}$ from Lemma 4.2 (where we take $\epsilon=\epsilon_{2}$ ). Taking $Q_{1, g}=B_{g}, \mathcal{D}_{1}=\mathcal{D}$, and $Y_{1, g}=Y_{g}$, we see that properties Z2) and Z3) for $\mathcal{Z}_{1}$ are satisfied. This form the base of induction. Notice that here we do not need to check the properties $\mathbf{Z 4 )}$ - Z6).

Now assume that the sets $\mathcal{Z}_{1} \supset \mathcal{Z}_{2} \supset \ldots \mathcal{Z}_{N}$ together with periodic orbits $\left\{Q_{1, g}, \ldots Q_{N, g}\right\},\left\{Y_{1, g}, \ldots Y_{N, g}\right\}$, functions $\gamma_{i}: \mathcal{Z}_{i} \rightarrow(0,+\infty)$, and sets $\mathcal{D}_{i}$ are constructed, $i=1, \ldots, N$. Let us construct the set $\mathcal{Z}_{N+1} \subset \mathcal{Z}_{N}$.

Notice that it is enough to construct an open dense subset of each connected component of $\mathcal{Z}_{N}$ (together with periodic orbits $Q_{N+1, g}, Y_{N+1, g}$, function $\gamma_{N+1}$, and a dense subset $\mathcal{D}_{N+1}$ ) that satisfies conditions $\mathbf{Z 2}$ ) - Z66). Take one of the connected components of the set $\mathcal{Z}_{N}$, denote it by $\mathcal{Z}^{*}$.

Let us enumerate diffeomorphisms from $\mathcal{D}_{N} \cap \mathcal{Z}^{*}=\left\{g_{i}\right\}_{i \in \mathbb{N}}$. Take one of these diffeomorphisms, say $g_{i} \in \mathcal{D}_{N} \cap \mathcal{Z}^{*}$.

Proposition 3.8 (applied to the saddles $Q_{N, g_{i}}$ and $Y_{N, g_{i}}$ ) allows to obtain a sequence of diffeomorphisms $g_{i k}, g_{i k} \rightarrow g_{i}$ as $k \rightarrow \infty$, such that each diffeomorphism $g_{i k}$ has a periodic saddle $S_{i k}$ with real multipliers (denoted by $R_{k}$ in Proposition (3.8), having the following properties: 
S1) the saddle $S_{i k}$ is homoclinically related to $Q_{N, g_{i k}}$, thus has the same index as $B_{g_{i}}$;

S2) for some constant $\Theta$ that does not depend on $k$ and for each $k \in \mathbb{N}$ we have $1<\left|\lambda^{F}\left(S_{i k}\right)\right|<\Theta ;$

S3) the map $g_{i k}$ has a heterodimensional cycle associated to $S_{i k}$ and $Y_{N, g_{i k}}$;

S4) take a real number $\gamma_{N}=\gamma_{N}\left(\mathcal{Z}^{*}\right)>0$ small enough (the choice of $\gamma_{N}$ will be clear from the construction below) and $\gamma_{N}$-neighborhoods $U_{\mathcal{O}\left(Y_{N, g_{i}}\right)}$ and $U_{\mathcal{O}\left(Q_{N, g_{i}}\right)}$ of the orbits of $Y_{N, g_{i}}$ and $Q_{N, g_{i}}$, and sequences of natural numbers $\ell_{k}, m_{k}$ that tend to infinity as $k \rightarrow \infty$, such that under the iterates of $g_{i k}$ it takes to the saddle $S_{i k}$ a fixed number of iterates (independent of $k$ ) to go from $U_{\mathcal{O}\left(Y_{N, g_{i}}\right)}$ to $U_{\mathcal{O}\left(Q_{N, g_{i}}\right)}$, then it remains $\ell_{k} \pi\left(Y_{N, g_{i}}\right)$ iterates in $U_{\mathcal{O}\left(Y_{N, g_{i}}\right)}$, then it needs a fixed number of iterates to go from $U_{\mathcal{O}\left(Y_{N, g_{i}}\right)}$ to $U_{\mathcal{O}\left(Q_{N, g_{i}}\right)}$, and finally it remains $m_{k} \pi\left(Q_{N, g_{i}}\right)$ iterates in $U_{\mathcal{O}\left(Q_{N, g_{i}}\right)}$. In particular, there is a constant $t \in \mathbb{N}$ independent of $k$ such that

$$
\pi\left(S_{i k}\right)=m_{k} \pi\left(Q_{N, g_{i}}\right)+\ell_{k} \pi\left(Y_{N, g_{i}}\right)+t .
$$

Exactly as in [15], properties (3) and (4) from Proposition 3.8 guarantee that making an arbitrary small perturbation of $g_{i k}$ (preserving properties $\mathbf{S 1}$ ) - S4)) we also have:

S5) the saddle $S_{i k}$ has the u-biaccumulation property.

We need the following quantitative estimates:

Lemma 4.4 (Lemma 5.4 from [15]). For every large $k \in \mathbb{N}$ the saddles $S_{i k}$ satisfy:

$$
\begin{gathered}
0<\chi^{F}\left(S_{i k}\right)<\frac{1}{2} \chi^{F}\left(Q_{N, g_{i k}}\right), \\
\frac{m_{k} \pi\left(Q_{N, g_{i}}\right)}{\pi\left(S_{i, k}\right)}=\frac{m_{k} \pi\left(Q_{N, g_{i}}\right)}{m_{k} \pi\left(Q_{N, g_{i}}\right)+\ell_{k} \pi\left(Y_{N, g_{i}}\right)+t}>1-C \chi^{F}\left(Q_{N, g_{i k}}\right) .
\end{gathered}
$$

Consider now the set

$$
\mathcal{D}^{\prime}=\left\{g_{i k} \mid S_{i k} \text { satisfies conditions (17), (8) }, i \geq 0 \text { and } k \geq 0\right\} \subset \mathcal{Z}^{*} \text {. }
$$

By construction, the set $\mathcal{D}^{\prime}$ is a countable dense subset of $\mathcal{Z}^{*}$. Let us enumerate the elements of $\mathcal{D}^{\prime}=\left\{h_{n}\right\}_{n \in \mathbb{N}}$. Let us also denote by $Q_{N+1, g}(n)$ the continuation of the periodic saddle $S_{i k}$ of the map $h_{n} \equiv g_{i k}$. In particular, $Q_{N+1, g_{i k}}(n)=S_{i k}$. 
Now an application of Lemma 3.5 and Proposition 3.7 implies that for each $h_{n}$ there is an open set $\mathcal{U}_{n}$, which is $\frac{1}{n}$-close to $h_{n}$, and for each $g \in \mathcal{U}_{n} \cap \mathcal{G}$ there is a saddle $Y_{N+1, g}(n)$ such that

Y1) the saddle $Y_{N+1, g}(n)$ is in $H(g)=H\left(P_{g}, g\right)$ and its orbit is $\epsilon_{N+2}$-dense in $H(g)$,

Y2) the saddles $Q_{N+1, g}(n)$ and $Y_{N+1, g}(n)$ have different indices,

Y3) the Lyapunov exponent $\chi^{F}\left(Y_{N+1, g}(n)\right)$ is close to $(-\nu)$, and

Y4) there is a dense subset $\widetilde{\mathcal{D}}(n)$ of maps in $\mathcal{U}_{n}$ having a heterodimensional cycle between $Q_{N+1, g}(n)$ and $Y_{N+1, g}(n)$.

We can take $\mathcal{U}_{n}$ small enough and close enough to $h_{n}$ to guarantee that for every $g \in \mathcal{U}_{n}$ one has $0<\chi^{F}\left(Q_{N+1, g}(n)\right)<\frac{1}{2} \chi^{F}\left(Q_{N, g}\right)$. Indeed, due to (7) this inequality holds for $h_{n}$. Since Lyapunov exponents of a hyperbolic saddle depend continuously on a diffeomorphism, the inequality holds also for all $g$ sufficiently $C^{1}$-close to $h_{n}$.

Let us now define inductively

$$
\mathcal{Z}(1)=\mathcal{U}_{1}, \mathcal{Z}(2)=\mathcal{U}_{2} \backslash \overline{\mathcal{Z}(1)}, \ldots, \mathcal{Z}(n)=\mathcal{U}_{n} \backslash \overline{\mathcal{Z}(n-1)}, \ldots
$$

and

$$
\mathcal{D}(n)=\widetilde{\mathcal{D}}(n) \cap \mathcal{Z}(n), n \in \mathbb{N} .
$$

Also we set

$$
\mathcal{Z}_{N+1}^{*}=\bigcup_{n \in \mathbb{N}} \mathcal{Z}(n), \quad \mathcal{D}_{N+1}^{*}=\bigcup_{n \in \mathbb{N}} \widetilde{\mathcal{D}}(n) .
$$

Besides, for each $g \in \mathcal{Z}(n)$ we set $Q_{N+1, g}=Q_{N+1, g}(n)$ and $Y_{N+1, g}=Y_{N+1, g}(n)$. Finally, we define $\mathcal{Z}_{N+1}$ and $\mathcal{D}_{N+1}$ as the union of constructed sets $\mathcal{Z}_{N+1}^{*}$ and $\mathcal{D}_{N+1}^{*}$ over all connected components of $\mathcal{Z}_{N}$. We claim that the set $\mathcal{Z}_{N+1}$ satisfies the required properties $\mathbf{Z 1}$ ) - Z6).

- Since the set $\left\{h_{n}\right\}_{n \in \mathbb{N}}$ is dense in $\mathcal{Z}^{*}$ (the chosen connected component of $\left.\mathcal{Z}_{N}\right)$, the union $\cup_{n \in \mathbb{N}} \mathcal{U}_{n}$ is dense in $\mathcal{Z}^{*}$, and hence $\cup_{n \in \mathbb{N}} \mathcal{Z}(n)$ is also dense in $\mathcal{Z}^{*}$, so $\mathbf{Z 1}$ ) holds.

- For each $g \in \mathcal{Z}(n)$ the saddle $Q_{N+1, g}(n)$ is homoclinically related to $B_{g}$ (recall S1)), has real multipliers, u-biaccumulation property, and $\pi\left(Q_{N, g}\right)<$ $\pi\left(Q_{N+1, g}(n)\right)$, so Z22) holds. 
- The sets $\widetilde{\mathcal{D}}(n) \subset \mathcal{U}_{n}$ and the saddles $Y_{N+1, g}(n)$ were constructed to satisfy Z3). More precisely, the estimate of the Lyapunov exponent $\chi^{F}\left(Y_{N+1, g}(n)\right)$ follows from the choice of $Y_{N+1, g}(n)$ in Y3), the $\epsilon_{N+2}$-density of the orbit of $Y_{N+1, g}(n)$ in $H(g)$ follows from $\left.\mathbf{Y} 1\right)$, and the existence of the heterodimensional cycle follows from $\mathrm{Y} 4)$.

- Take $g \in \mathcal{Z}_{N+1}$, and denote by $\Gamma$ the part of the orbit of $Q_{N+1, g}$ that belongs to the neighborhood $U_{Q_{N, g}}$. Define the projection

$$
\rho: \Gamma \rightarrow \mathcal{O}\left(Q_{N, g}\right), \quad \rho(x)=\left\{\text { the point of } \mathcal{O}\left(Q_{N, g}\right) \text { nearest to } x\right\} .
$$

By construction (recall S4)),

$$
\# \Gamma=m_{k} \pi\left(Q_{N, g}\right) \quad \text { and } \quad \#\left(\mathcal{O}\left(Q_{N+1, g}\right)\right)=m_{k} \pi\left(Q_{N, g}\right)+\ell_{k} \pi\left(Y_{N, g}\right)+t .
$$

Recall that here $k$ and $n$ are related due to the enumeration $h_{n}=g_{i k}$; notice that in fact integers $m_{k}, \ell_{k}$, and $t$ depend also on the index $i$, but our notations do not reflect this dependence. Now the first part of $\mathbf{Z 4}$ ) follows from the inequality (8). The second part, $B_{\epsilon_{N+2}}\left(\mathcal{O}\left(Q_{N+1, g}\right)\right) \supset \mathcal{O}\left(Y_{N, g}\right)$, follows from the fact that $\gamma_{N}$ can be chosen arbitrarily small, in particular, smaller than $\epsilon_{N+2}$.

- Condition Z5) is obtained by choosing sufficiently small $\gamma_{N}>0$.

- The last property Z6) follows directly from inequality (7).

We completed the step of induction, and, thus, proved Proposition 4.3.

\subsection{Infinite sequences of periodic orbits}

Here we conclude the proof of Theorem 2 and, therefore, of the main result.

Proposition 4.5. Let $f$ satisfy the conditions of Theorem Q Arbitrary close to $f$ there exists an open set $\mathcal{Z} \subset \operatorname{Diff}^{1}(M)$ such that generic diffeomorphism $g$ from $\mathcal{Z}$ have a sequence of periodic saddles which satisfies the assumptions of Proposition 2.7, belongs to the homoclinic class $H\left(P_{g}, g\right)$, and the union of their orbits is dense in the homoclinic class $H\left(P_{g}, g\right)$.

Note that Propositions 4.5 and 2.7 give non-hyperbolic ergodic measures supported on the whole homoclinic class for generic diffeomorphisms from $\mathcal{Z}$, and, thus, Theorem 2 . 
Proof. Apply Proposition 4.3, and set $\mathcal{Z}=\mathcal{Z}_{1}$. Due to Property Z1), for any $N \in \mathbb{N}$ the set $\mathcal{Z}_{N}$ is an open and dense subset of $\mathcal{Z}$. Therefore the intersection

$$
\mathcal{R}=\mathcal{G} \cap\left(\bigcap_{N \in \mathbb{N}} \mathcal{Z}_{N}\right)
$$

is a residual subset of $\mathcal{Z}$.

Take any $g \in \mathcal{R}$. Due to $\mathbf{Z 2}$ ), for the diffeomorphism $g \in \mathcal{R}$ a sequence of periodic points

$$
\left\{B_{g}, Q_{1, g}, Q_{2, g} \ldots, Q_{N, g}, \ldots\right\} \subset H\left(P_{g}, g\right)
$$

is well defined. We claim that this sequence satisfies the assumptions of Proposition 2.7.

Indeed, assumption $\mathbf{1}$ ) holds since $g \in \mathcal{G}$ and due to the choice of $\mathcal{Z}$ (existence of a one dimensional center direction for $\Delta=H\left(P_{g}, g\right)$ ), and 2) follows from property Z2). Assumptions 3), 4), and 5) follow from Z4), Z5), and Z6), respectively.

Finally, since $\epsilon_{N} \rightarrow 0$ as $N \rightarrow \infty$, from Z3), Z4) we have that the nonhyperbolic ergodic measure given by Proposition 2.7] satisfy

$$
\operatorname{supp}(\mu)=\bigcap_{N \in \mathbb{N}}\left(\overline{\bigcup_{K \geq N} \mathcal{O}\left(Q_{K, g}\right)}\right)=H\left(P_{g}, g\right)
$$

This proves Proposition 4.5.

\section{References}

[1] F. Abdenur, Ch. Bonatti, S. Crovisier, Nonuniform hyperbolicity for $C^{1}$ generic diffeomorphisms, arXiv:0809.3309v1.

[2] F. Abdenur, Ch. Bonatti, S. Crovisier, L. J. Díaz, L. Wen, Periodic points and homoclinic classes, Ergodic Th. and Dynam. Syst., 26, (2006), pp 1-22,

[3] F. Abdenur, L. J. Díaz, Pseudo-orbit shadowing in the $C^{1}$-topology, Discrete Contin. Dyn. Syst., 17 (2007), pp. 223-245.

[4] M. Asaoka, Hyperbolic sets exhibiting $C^{1}$-persistent homoclinic tangency for higher dimensions, Proc. Amer. Math. Soc., 136 (2008), pp. 677-686. 
[5] Ch. Bonatti, L. J. Díaz, Nonhyperbolic transitive diffeomorphisms, Ann. of Math., 143 (1996), pp. 357-396.

[6] Ch. Bonatti, L. J. Díaz, Robust heterodimensional cycles, J. Inst. Math. Jussieu, 7 (2008), pp. 469-525.

[7] Ch. Bonatti, L. J. Díaz, T. Fisher, Super-exponential grothw of the number of periodic orbits inside homoclinic classes, Discrete Contin. Dyn. Syst., 20 (2008), pp. 589-604.

[8] Ch. Bonatti, L. J. Díaz, E. R. Pujals, A $\mathcal{C}^{1}$-generic dichotomy for diffeomorphisms: Weak forms of hyperbolicicity or infinitely many sinks or sources, Ann. of Math., 158 (2003), pp 355-418.

[9] Ch. Bonatti, L. J. Díaz, G. Turcat, Pas de "Shadowing Lemma" pour les dynamiques partiellement hyperboliques, C. R. Acad. Sci., Paris, Sr. I, Math., 330 (2000), pp. 587-592.

[10] Ch. Bonatti, L.J. Díaz, and M. Viana, Dynamics beyond uniform hyperbolicity Encyclopaedia of Mathematical Sciences (Mathematical Physics), 102, Springer Verlag, (2005).

[11] J. Bochi, M. Viana, Uniform (projective) hyperbolicity or no hyperbolicity: a dichotomy for generic conservative maps, Ann. Inst. H. Poincaré, Anal. Non Linéaire, 19 (2002), pp. 113-123.

[12] M. Carvalho, Sinai-Ruelle-Bowen measures for $N$-dimensional derived from Anosov diffeomorphisms, Ergodic Th. and Dynam. Syst., 13 (1993), pp. 21-44.

[13] C. Carballo, C. Morales, M. J. Pacifico, Homoclinic classes for generic $C^{1}$ vector fields, Ergodic Th. and Dynam. Syst., 23 (2003), pp. 403-415.

[14] L. J. Díaz, Robust non-hyperbolic dynamics at heterodimensional cycles, Ergodic Th. and Dynam. Syst., 15 (1995), pp. 291-315.

[15] L. J. Díaz, A. Gorodetski, Non-hyperbolic invariant measures for nonhyprerbolic homoclinic classes, Ergodic Theory Dynam. Systems, to appear.

[16] L. J. Díaz, T. Fisher, Symbolic extensions for partially hyperbolic diffeomorphisms, arXiv:0906.2176. 
[17] L. J. Díaz, J. Rocha, Partially hyperbolic and transitive dynamics generated by heteroclinic cycles, Ergodic Th. and Dynam. Syst., 21 (2001), pp. 25-76.

[18] T. Downarowicz, S. Newhouse, Symbolic extensions and smooth dynamical systems, Invent. Math., 160 (2005), pp. 453-499.

[19] A. Gorodetski, Regularity of central leaves of partially hyperbolic sets and its applications, Izvestia RAN (The Newsletter of the Russian Academy of Science), 70 (2006), pp. 52-78.

[20] A. Gorodetski, Yu. Ilyashenko, Some new robust properties of invariant sets and attractors of dynamical systems, Functional Analysis and Applications, 33 (1999), pp. 16-30.

[21] A. Gorodetski, Yu. Ilyashenko, V. Kleptsyn, M. Nalsky, Nonremovable zero Lyapunov esponents, Functional Analysis and Its Applications, 39 (2005), pp. 27-38.

[22] N. Gourmelon, Instabilité de la dynamique en l'absence de décomposition dominée, Ph. D. Thesis Univ. de Bourgogne 2006.

[23] S. Hayashi, Connecting invariant manifolds and the solution of the $C^{1}$ stability and $\Omega$-stability conjectures for flows, Ann. of Math., 145 (1997), pp. $81-137$.

[24] V. Kaloshin, Generic diffeomorphisms with superexponential growth of number of periodic points, Comm. in Math. Physics, 211 (2000), pp. 253271.

[25] V. Kleptsyn, M. Nalsky, Stability of the existence of nonhyperbolic measures for $C^{1}$-diffeomorphisms, (Russian) Funktsional. Anal. i Prilozhen. 41 (2007), no. 4, pp. 30-45, 96; translation in Funct. Anal. Appl. 41 (2007), no. 4 , pp. $271-283$.

[26] R. Mañé, Contributions to the stability conjecture, Topology, 17 (1978), pp. 383-396.

[27] R. Mañé, Ergodic theory and differentiable dynamics. Ergebnisse der Mathematik und ihrer Grenzgebiete. 3. Folge, Bd. 8., Springer-Verlag, (1987). 
[28] M. Nalsky, Non-hyperbolic invariant measures on a maximal attractor, arXiv: 0807.4963v1.

[29] S. Newhouse, Hyperbolic Limit Sets, Trans. AMS., 167 (1972), pp. 125-150.

[30] V. I. Oseledec, A multiplicative ergodic theorem. Characteristic Lyapunov exponents of dynamical systems. (Russian) Trudy Moskov. Mat. Ob., 19 (1968), pp. 179-210.

[31] J. Palis, A global view of dynamics and a conjecture on the denseness of finitude of attractors, Astérisque, 261 (2000), pp. 335-347.

[32] C. Pugh, An improved closing lemma and a general density theorem, Amer. J. Math., 89 (1967), pp. 1010-1021.

[33] D. Ruelle, A. Wilkinson, Absolutely singular dynamical foliations, Comm. Math. Phys., 219 (2001), pp. 481-487.

[34] K. Sakai, $C^{1}$-stable shadowing chain components, Ergodic Th. and Dynam. Syst., 28 (2008), pp. 987-1029.

[35] M. Shub, A. Wilkinson, Pathological foliations and removable zero exponents, Invent. Math., 139 (2000), pp. 495-508.

[36] J. Yang, $C^{1}$-dynamics far from tangencies, PhD Thesis IMPA (2008).

[37] G. -C. Yuan, J. A. Yorke, An open set of maps for which every point is absolutely nonshadowable, Proc. Amer. Math. Soc., 128 (2000), pp. 909918.

Christian Bonatti (bonatti@u-bourgogne.fr)

Institut de Mathématiques de Bourgogne

B.P. 47870

21078 Dijon Cedex

France 
Lorenzo J. Díaz (lodiaz@mat.puc-rio.br)

Departamento de Matemática, PUC-Rio

Marquês de S. Vicente 225

22453-900 Rio de Janeiro RJ

Brazil

Anton Gorodetski (asgor@math.uci.edu)

Department of Mathematics

University of California, Irvine

Irvine, CA 92697

USA 